\title{
High efficiency tapered free-electron lasers with a prebunched electron beam
}

\author{
C. Emma, ${ }^{1}$ N. Sudar, ${ }^{1}$ P. Musumeci, ${ }^{1}$ A. Urbanowicz, ${ }^{1}$ and C. Pellegrini ${ }^{1,2}$ \\ ${ }^{1}$ University of California, Los Angeles, California 90095, USA \\ ${ }^{2}$ SLAC National Accelerator Laboratory, Menlo Park, California 94025, USA \\ (Received 5 September 2017; published 17 November 2017; corrected 1 February 2018)
}

\begin{abstract}
In this paper we analyze the high gain, high efficiency tapered free-electron laser amplifier with a prebunched electron beam. Simple scaling laws are derived for the peak output power and extraction efficiency and verified using 1D simulations. These studies provide useful analytical expressions which highlight the benefits resulting from fine control of the initial conditions of the system, namely the initial electron beam bunching and input seed radiation. When time-dependent effects are included, the sideband instability is known to limit the radiation amplification due to particle detrapping. We discuss two different approaches to mitigate the sideband growth via 1-D time dependent simulations. We find that a more aggressive taper enabled by strong prebunching and a modulation of the resonance condition are both effective methods for suppressing the sideband instability growth rate.
\end{abstract}

DOI: 10.1103/PhysRevAccelBeams.20.110701

\section{INTRODUCTION}

Efficient generation of tunable, coherent, high power radiation can enable revolutionary applications in a wide range of fields from power beaming [1,2] to extreme ultraviolet lithography $[3,4]$ to $\mathrm{x}$-ray science [5-7]. The free-electron laser (FEL) amplifier has naturally been considered as a candidate to reach high peak and average power for wavelength ranges lacking a suitable solid-state (or gasphase) gain medium. In terms of efficiency, this approach is limited as high gain FELs typically operate in the linear exponential gain regime [8]. Saturation occurs as soon as the wave intensity reaches a level for which the electrons exchange a significant amount of energy so that they start dephasing in the ponderomotive potential bucket within a radiation gain length. The saturation efficiency, given by the Pierce parameter $\rho$ [8], is typically limited to $\sim 0.1 \%$.

A solution to this problem is obtained if the undulator is tapered in such a way that the phase of the electrons is kept constant within the wave even as their energy is being modified by the interaction with the radiation. This is the nonlinear regime of the FEL interaction which holds the promise for very efficient energy extraction [9-11]. General analytical predictions in the nonlinear regime are hard to obtain and one typically relies on numerical simulations in order to predict the dynamics of the system. The subject has been at the center of intense studies as undulator tapering opens the door to very high peak and average power

Published by the American Physical Society under the terms of the Creative Commons Attribution 4.0 International license. Further distribution of this work must maintain attribution to the author(s) and the published article's title, journal citation, and DOI. radiation sources both in the $\mathrm{x}$-ray regime and at longer wavelengths [12-15].

Specifically, numerical optimization studies have shown that with a judiciously chosen taper profile, the output efficiency of a seeded FEL can be increased by two orders of magnitude to $\sim 10 \%[10,11]$. At longer wavelengths, a recent experiment has demonstrated 30\% energy extraction from a prebunched electron beam interacting with a large external laser seed in a strongly tapered undulator [16]. It is therefore interesting to study from a theoretical point of view the effect of prebunching on high efficiency tapered FELs. In this paper we limit the discussion to the onedimensional regime, so that analytical expressions for the output power could be obtained for the most general initial conditions. The results, validated using a fast Matlab-based 1D FEL code [17], highlight the importance of an intense input seed power level and of prebunching the initial longitudinal beam distribution.

One of the main concerns in the nonlinear FEL regime is related to the evolution of the radiation spectrum. Early studies of tapered FELs uncovered the deleterious effects of sideband radiation growth due to the synchrotron oscillation motion of the particles in the longitudinal phase space $[18,19]$. The resulting chaotic motion is responsible for particle detrapping and loss of efficiency in the radiation amplification [20]. We therefore took advantage of the computational speed increase enabled by the $1 \mathrm{D}$ approximation to analyze a variety of schemes to compensate this effect.

It should be stressed here that three dimensional effects are certainly poised to have a dominant impact on the final behavior of high gain high efficiency FELs $[13,15,21,22]$. Reference [15] for example discusses an analytical model for tapering optimization including diffraction, however it 
assumes a small seed and an unbunched electron beam. Diffraction and beam emittance will change quantitavely, but not qualitatively, many of the results presented. Nevertheless, our 1D analysis uncovers the strong benefits resulting from controlling the beam and radiation initial conditions and tests the effectiveness of compensation schemes for the sideband instability.

The paper is organized as follows: we first discuss the time-independent high gain high efficiency regime obtaining a useful power scaling law. Subsequently, we analyze and compare the estimates provided by the power scaling with 1-D simulations starting from different initial conditions. We then look at the time-dependent cases and discuss sideband instability mitigation measures. The discussion concludes by considering the implications of this work to future high efficiency high gain FEL studies.

\section{1D EFFICIENCY SCALING FOR A HIGH GAIN HIGH EFFICIENCY FEL}

The 1-D FEL equations for a helical undulator can be written as $[23,24]$ :

$$
\begin{aligned}
\frac{d \psi_{j}}{d z} & =k_{w} \times\left(1-\frac{\gamma_{r}^{2}}{\gamma_{j}^{2}}\right) \\
\frac{d \gamma_{j}}{d z} & =-\frac{\chi_{1} K E}{\gamma_{j}} \sin \psi_{j} \\
\frac{d E}{d z} & =\chi_{2} K\left\langle\frac{\sin \psi_{j}}{\gamma_{j}}\right\rangle
\end{aligned}
$$

with $\chi_{1}=e / m_{e} c^{2}, \chi_{2}=Z_{0} I / 4 \pi \sigma_{e}^{2}$ and $Z_{0}=1 / c \epsilon_{0}$. The undulator is described by the parameter $K=e B / m c k_{w}$ and the wave number $k_{w}$ and the radiation field by the amplitude $E$ and wave number $k$. The electron beam has peak current $I$ and rms spot size $\sigma_{e}$, and the resonant energy is defined as $\gamma_{r}^{2}=k / 2 k_{w} *\left(1+K^{2}\right)$. An important quantity in the context of tapered FELs is the resonant phase, the phase of the particle at the resonant energy, which can be written as:

$$
\sin \psi_{r}=\frac{k}{2 k_{w}} \frac{\left|K^{\prime}\right|}{E}
$$

where $K^{\prime}$ is the rate of change of the undulator field. The choice of resonant phase affects both the rate at which the undulator is tapered and the size of the stable bucket in which trapped electrons continue to lose energy to the radiation field [9]. In the resonant particle approximation where $\gamma_{j} \approx \gamma_{r}$ and $\sin \psi_{j} \approx \sin \psi_{r}$, the efficiency is given by:

$\eta=\frac{1}{\gamma_{0}}\left|\sum_{j} \gamma_{j}(z)-\gamma_{j, 0}\right| \approx f_{t}\left|\frac{\gamma_{r}(z)-\gamma_{0}}{\gamma_{0}}\right|=f_{t}\left|\frac{\Delta \gamma_{r}}{\gamma_{0}}\right|$ where $f_{t}$ is the fraction of trapped electrons which in general depends on the size of the bucket, i.e., the input seed power, the undulator field and the resonant phase. Note that we assume for simplicity that the trapping fraction and the resonant phase are independent of $z$ in the post-saturation regime.

Different initial conditions for tapered FELs result in different trapping fractions and different scaling of the output efficiency. Keeping $f_{t}$ as general for the moment we can write the change in resonant energy using Eq. (2):

$$
\Delta \gamma_{r}=-\frac{\chi_{1} K_{0}}{\gamma_{0}} \sin \psi_{r} \int E\left(z^{\prime}\right) d z^{\prime}
$$

where we assume $K / \gamma \approx K_{0} / \gamma_{0}$, which is a good approximation for small efficiencies. From Eq. (3) we can calculate the radiation field evolution, which in the 1-D limit with a constant resonant phase is linear in $z$ :

$$
E \approx E_{0}+\frac{K_{0}}{\gamma_{0}} f_{t} \chi_{2} \sin \psi_{r} z
$$

with $E_{0}$ denoting the seed field. Putting together Eqs. (5)-(7) we have an approximation for the efficiency:

$$
\eta=\frac{\chi_{1} f_{t}}{\gamma_{0}}\left[E_{0} \frac{K_{0}}{\gamma_{0}} \sin \psi_{r} z+\frac{f_{t} \chi_{2}}{2}\left(\frac{K_{0}}{\gamma_{0}} \sin \psi_{r} z\right)^{2}\right]
$$

and with $P_{\text {rad }}=P_{0}+\eta P_{\text {beam }}$, after rearranging the constants we get:

$P_{\mathrm{rad}}=[P_{0}+\underbrace{E_{0}\left(\frac{K_{0}}{\gamma_{0}}\right) \bar{I} \sin \psi_{r} z}_{P_{1}}+\underbrace{\frac{Z_{0}}{8 \pi \sigma_{e}^{2}}\left(\frac{K_{0}}{\gamma_{0}}\right)^{2}\left(\bar{I} \sin \psi_{r} z\right)^{2}}_{P_{2}}]$

where $\bar{I} \equiv f_{t} I$ is the trapped particle current and the seed power is given by $P_{0}=2 \pi \sigma_{e}^{2} E_{0}^{2} / Z_{0}$. Note that in the absence of an input seed we recover the familiar scaling for coherent emission from a bunched beam $P_{\text {rad }}=P_{2} \propto(\bar{I} z)^{2}$. This is also true for long undulators since the quadratic term $P_{2}$ dominates the radiation power scaling if the number of undulator periods satisfies $N_{u} \gg \frac{\sqrt{32 \pi / Z_{0}}}{\sin \psi_{r}} \frac{\gamma_{0}}{K_{0}} \frac{\sqrt{P_{0}}}{I} \frac{\sigma_{e}}{\lambda_{w}}$. At the same time for short undulators and intense seed pulses, the efficiency and output power are linearly proportional to the undulator length and the field strength. This is the low gain tapering enhanced stimulated superradiant amplification regime [10]. It is important to recognize, as originally suggested by KMR and revisited in recent studies $[9,13,14]$, the advantages of a variable $\psi_{r}(z)$ on the output efficiency. Optimization of the resonant phase variation, beyond the scope of this work, is often carried out numerically and is set to increase the output power for both unbunched and prebunched electron 
TABLE I. 1D simulation parameters for the tapered FEL cases shown in Secs. II A-B.

\begin{tabular}{lc}
\hline \hline Parameter & Value \\
\hline Beam Energy [GeV] & 4.5 \\
Peak Current [kA] & 4 \\
Relative Energy Spread & $\rho / 6$ \\
Transverse beam size $[\mu \mathrm{m}]$ & 20 \\
Photon Energy [keV] & 1 \\
Undulator Period [cm] & 2 \\
Undulator Parameter K $(\mathrm{rms})$ & 3 \\
FEL parameter $\rho\left[\times 10^{-3}\right]$ & 2 \\
\hline \hline
\end{tabular}

beams. In the following sections we analyze the power scaling as it applies to a tapered FEL amplifier starting from a large seed with an unbunched and a prebunched electron beam.

\section{A. The large seed, unbunched beam case}

The simplest case to treat is that starting from a large seed with a cold unbunched beam. In this case the trapping fraction can be approximated by the bucket width $f_{t}=$ $f_{b}=\frac{\psi_{2}-\psi_{1}}{2 \pi}$ with $\psi_{2}=\pi-\psi_{r}$ and $\psi_{1}$ the solution of the equation:

$$
\cos \psi_{1}+\psi_{1} \sin \psi_{r}=\cos \psi_{2}-\psi_{2} \sin \psi_{r}
$$

The trapping fraction is then a function of the resonant phase only and not the input seed power. We compare the analytical power estimate from Eq. (9) with numerical integration of the 1-D FEL equations starting from a seed power $P_{0}=1.6 \rho P_{\text {beam }}$ and scanning the resonant phase in the range $0<\psi_{r}<90^{\circ}$. The simulation parameters are given in Table I and the results shown in Fig. 1. The

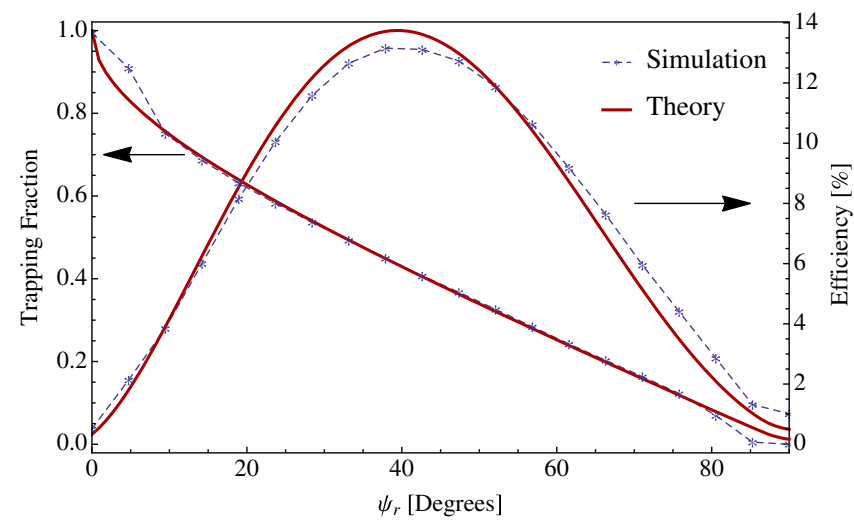

FIG. 1. Power transfer efficiency and trapping fraction for a seeded tapered FEL amplifier at constant resonant phase. The seed power is $1.6 \rho P_{\text {beam }}$ and the taper starts at $z=0$ for the $N_{u}=10^{3}$ period undulator. The theory estimate [Eq. (9)] is in good agreement with simulation and the trapping fraction is accurately given by the bucket width. Arrows show the axes belonging to the curves. analytical efficiency estimate matches the simulation results well with the maximum efficiency reaching $13 \%$ in this configuration. Note that in the simulation results shown in Fig. 1 the trapping fraction values stay constant throughout the undulator. We observe that this recovers the known result of Ref. [25] in the low gain (constant $P_{\text {rad }}$ ) high efficiency FEL for which the optimal resonant phase is also given by $\psi_{r} \approx 40^{\circ}$ for a cold beam with $f_{t}=f_{b}$. This occurs because the efficiency in the low and high gain cases scales as $\left(f_{t} \sin \psi_{r}\right)$ and $\left(f_{t} \sin \psi_{r}\right)^{2}$ respectively, and is maximized at the same value of $\psi_{r}$. For completeness we mention that in the limit of a warm beam, with energy spread comparable to the bucket size, the trapping fraction scales as the bucket area $\left(f_{t} \propto \alpha\left(\psi_{r}\right) \approx\right.$ $\left(1-\sin \psi_{r}\right) /\left(1+\sin \psi_{r}\right)$ [26]) which decreases faster than the bucket width as a function of $\psi_{r}$. Consequently, the peak efficiency is reduced and the optimal resonant phase is smaller, around $\psi_{r} \approx 20^{\circ}$ [25].

\section{B. The large seed, prebunched beam case}

From Eq. (9) for the power estimate we see that increasing the trapping fraction greatly increases the output power for the same resonant phase. One method for maximizing the fraction of particles trapped in the bucket is to inject a prebunched electron beam, for example by using a single stage or two-stage prebuncher setup similar to that described in Appendix B and discussed in Refs. [27,28]. The trapping fraction in this case (see Fig. 2) is not only a function of the resonant phase but also of the initial laser seed power expressed via the modulation parameter $A \equiv h_{b} / \sigma_{\gamma}$ which is the ratio between the bucket height at zero resonant phase $h_{b}=$ $\sqrt{2 \chi_{1} K E_{0} / k_{w}}$ and the initial rms electron slice energy spread. Figure 2 shows the analytic fit of the trapping fraction $f_{t}\left(A, \psi_{r}\right)$ for optimal buncher settings in a single or double-buncher configuration with a bucket height 10-30 times larger than the initial electron energy spread. The increased trapping compared to the unbunched case (Fig. 1) helps mitigate the trade-off between deceleration gradient $\left(P_{\text {rad }} \propto \sin ^{2} \psi_{r}\right)$ and particle loss $\left(P_{\text {rad }} \propto f_{t}^{2}\right)$ which is central to the optimization of tapered FEL systems. The advantage of prebunching becomes clearer when we directly compare the efficiency for a modest modulation strength $A=10$ in the double-buncher case to the unbunched case (see Fig. 3). The efficiency peaks at $\psi_{r} \approx 45^{\circ}$ compared to the unbunched case at $\psi_{r} \approx 40^{\circ}$, and its peak value is around a factor of 2.5 larger. Note that the analytic formula matches simulation well for efficiencies $\eta \leq 40 \%$, with the peak efficiency for the $A=30$ case reaching up to $50 \%$. At larger values, the approximation $K / \gamma \approx K_{0} / \gamma_{0}$ and the assumption of constant trapping fraction break down as the bucket height starts to decrease toward the end of the undulator and electrons begin to detrap. 

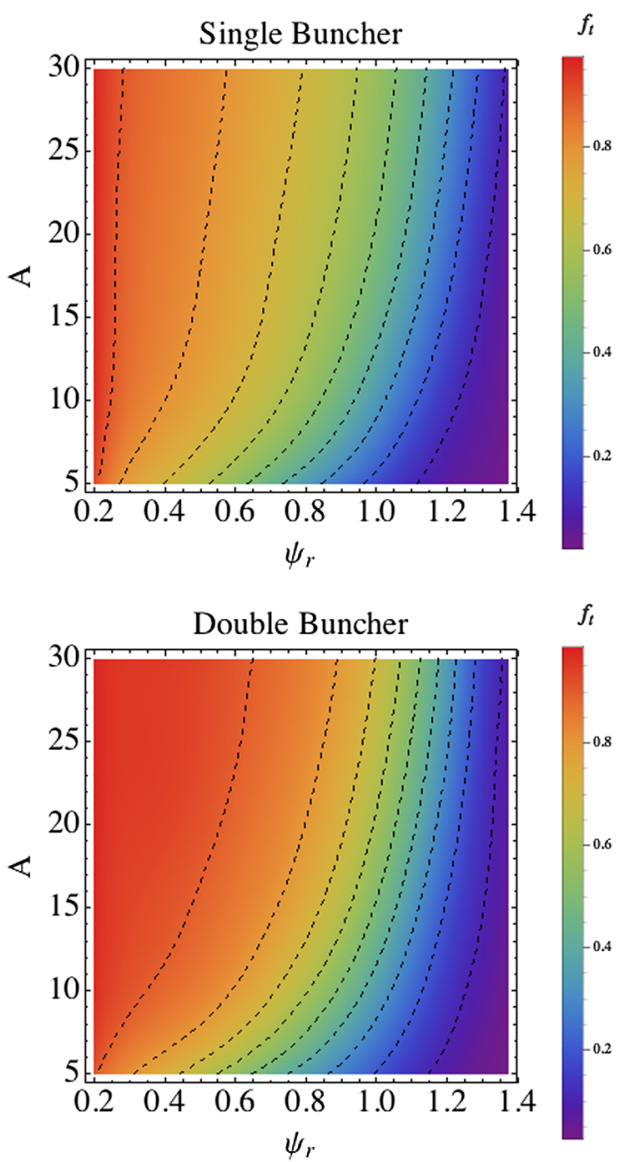

FIG. 2. Analytic fit of the trapping fraction $f_{t}$ as a function of the modulation parameter $A \equiv h_{b} / \sigma_{\gamma}$ (ratio of the modulation bucket height and the electron slice energy spread) and the resonant phase between $\pi / 16-7 \pi / 16$. The trapping fraction obtained with pre-bunching is larger than in the unbunched case leading to higher power transfer efficiency (see Fig. 3). The advantage of the double buncher compared to the single buncher scheme (see Appendix B) is largest for A $>10$ and $\psi_{r}<\pi / 4$.

The main challenge in realizing this scheme would be to generate the seed laser power capable to induce an $A=10$ modulation. This could be achieved for example in a fresh bunch configuration [29-31] where the first bunch is used to generate the modulation power and then discarded while the second bunch is overlapped with the seed in a single or double-buncher section before entering the tapered amplifier downstream (see Appendix B). As shown in Fig. 2-3 larger modulations $(A=20-30)$ generate larger trapping fractions and maximize the efficiency with a more aggressive taper at a larger resonant phase $\left(\psi_{r}=45^{\circ}-55^{\circ}\right)$. The advantage of tapering the undulator at larger resonant phase is a faster energy extraction and higher peak power in a shorter undulator length. As we discuss in the next section, this will reduce the sensitivity to the parasitic time dependent effects of the sideband instability.

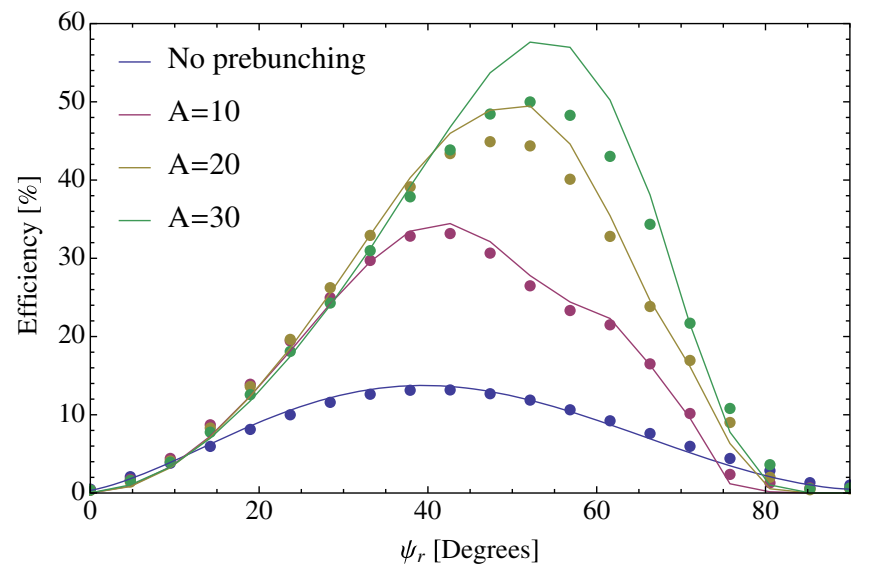

FIG. 3. Analytic estimate (solid) and simulation (dots) of the efficiency with/without pre-bunching for three different values of the modulation strength A and the parameters of Table I. The analytic formula is in good agreement for values of the efficiency $\eta<40 \%$. For $\eta>40 \%$ the assumption of constant trapping fraction breaks down as particles begin to detrap due to the bucket height $\left(h_{b} \propto \sqrt{K E}\right)$ decreasing towards the end of the undulator.

\section{1-D THEORY: TIME DEPENDENT}

\section{A. Sideband instability scaling with resonant phase}

Time dependent effects in tapered FELs are known to generate instabilities which can reduce the efficiency of the system. The most deleterious effects for a high gain, high efficiency FEL are due to the synchrotron sideband instability. The sideband instability results from the resonance between the electron synchrotron motion and sideband radiation shifted from the resonant wavelength by an amount $\Delta \lambda / \lambda= \pm \lambda_{w} / L_{s}$, where $\lambda_{w}$ is the undulator period and $L_{s}$ is the synchrotron oscillation period:

$$
\frac{L_{s}}{\lambda_{w}}=\sqrt{\frac{k}{4 \chi_{1} E \cos \psi_{r}} \frac{1+K^{2}}{K} .}
$$

We study the growth rate of the sideband instability at a constant resonant phase following the original work done by KMR. In a simple low gain two-frequency system, the gain of the sideband field $E_{s}(z)$ relative to the primary wave $E(z)$ is given by [18]:

$$
G \equiv \frac{E}{E_{s}} \frac{E_{s}^{\prime}}{E^{\prime}} \approx \frac{N_{s}}{4 \sin 2 \psi_{r}}
$$

where $N_{s}$ is the number of synchrotron oscillations performed by the bunch throughout the undulator. As such the scaling $G \propto 1 / \sin 2 \psi_{r}$ predicts significant sideband suppression at increasing resonant phase. Qualitatively this can be understood as in a high gain tapered amplifier, the sidebands are suppressed more strongly than in the 

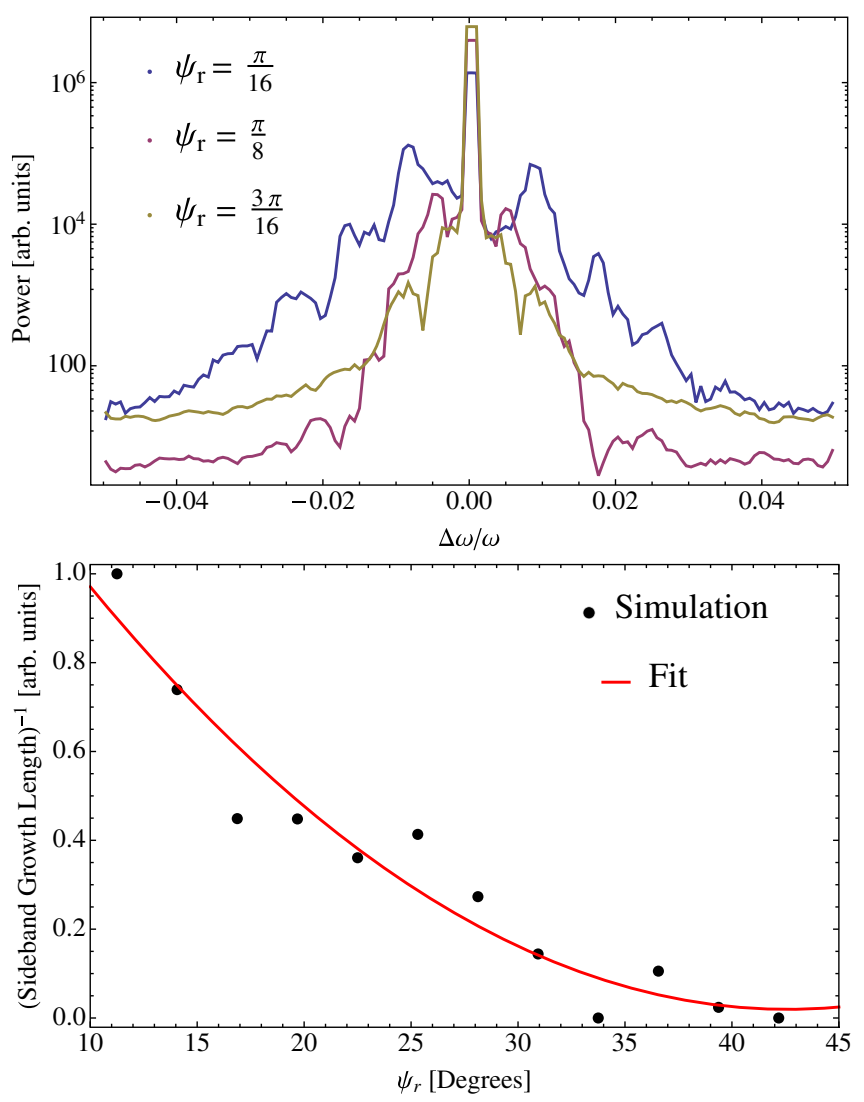

FIG. 4. (top) Spectra from 1-D time dependent simulations of a tapered FEL amplifier at constant resonant phase. The reduction in sideband intensity with resonant phase and the larger sideband growth length (bottom) reflects the scaling of the sideband gain $G \propto 1 / \sin 2 \psi_{r}$ from Ref. [18].

low-gain case since the electric field, and thus the synchrotron frequency, are changing rapidly. This damps the resonant interaction between the synchrotron oscillation and the sideband radiation. At larger resonant phase the deceleration gradient is larger and the synchrotron frequency varies more rapidly. It is therefore advantageous to taper the FEL using a large resonant phase. We show an example of this sideband suppression via 1-D time dependent simulations in Fig. 4 for the same parameters as in Table I and three different values of the resonant phase.

The advantage of having a prebunched electron beam with regards to the sideband instability is twofold. First, the increasing gain speeds up the variation of the synchrotron frequency at any resonant phase compared to the unbunched case. This prevents the sideband frequencies from resonating effectively with the electron synchrotron motion and reduces the sideband amplification. Second, one achieves increased sideband suppression due to the optimal resonant phase being larger than in the unbunched case (see Fig. 3). This second fact is especially important considering that when 3-D effects are included, radiation diffraction will tend to reduce the field amplitude to an asymptotic value [21]. An asymptotic value for the field implies a nearly constant synchrotron frequency and consequently large sideband growth [11]. Thus enhanced energy extraction in the shortest distance enabled by prebunching (large $\psi_{r}$ ) is important for a high efficiency tapered FEL amplifier.

\section{B. Sideband suppression via gain modulation}

Suppressing the sideband instability can be achieved, for example, by using phase shifters to generate destructive interference at the sideband frequencies, with the drawback that this method requires isochronous chicanes for successful implementation [10]. Alternatively, one can circumvent the sideband problem by carefully selecting the initial conditions such that the radiation seed is large enough to vastly exceed the shot noise power which triggers the sideband growth $[11,30]$. We present an alternative sideband suppression method by using the undulator field to modulate the gain of the FEL and induce a phase shift on the radiation to ensure destructive interference at the first harmonic sideband wavelength $\lambda=\lambda_{0}\left(1 \pm \lambda_{w} / L_{s}\right)$.
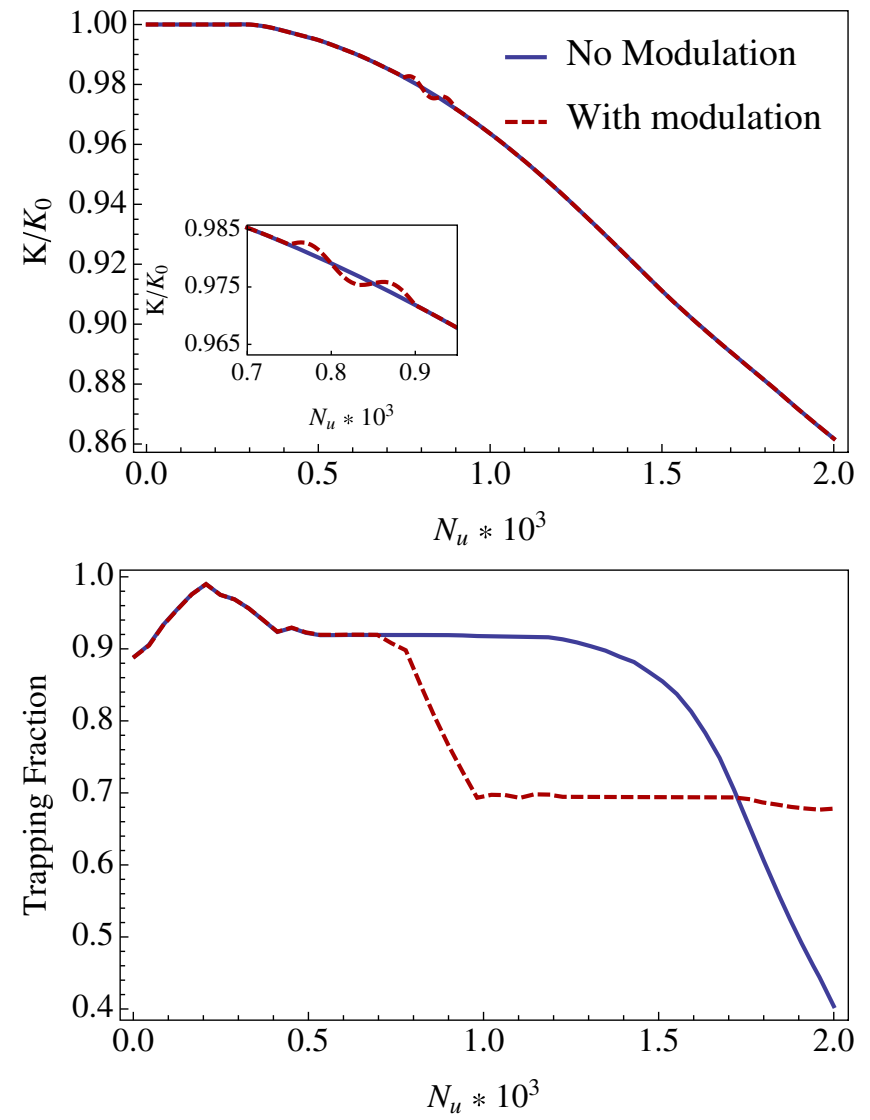

FIG. 5. (Top) Undulator taper profile for a gain modulated tapered FEL. The modulation section at $N_{u}=750$ changes the synchrotron frequency and damps the sideband growth (see Fig. 6). (Bottom) The trapping fraction drops after the modulation section but remains constant compared to the unmodulated case which suffers from severe sideband-induced detrapping after $N_{u}=1500$. 

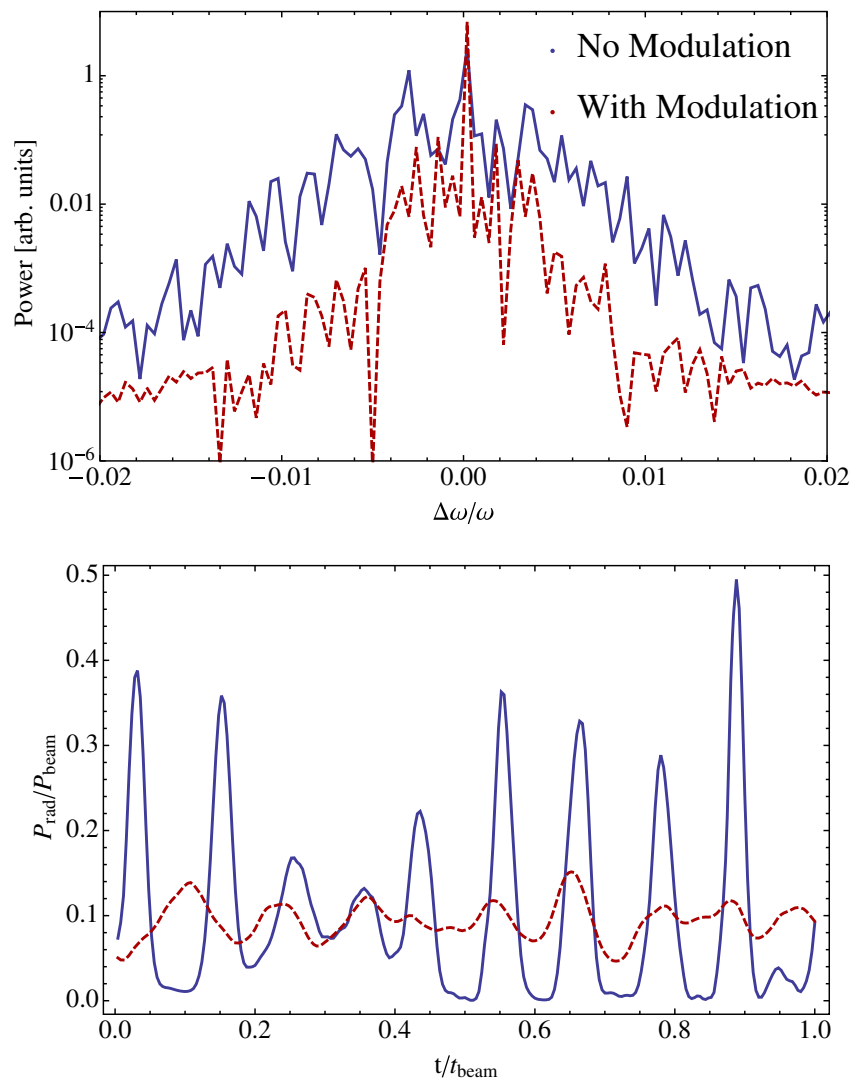

FIG. 6. Radiation spectrum (top) and temporal profile (bottom) with and without gain modulation showing sideband reduction for a gain modulated high efficiency FEL. The ratio of sideband to total power is $55 \%$ in the unmodulated case and $4 \%$ in the modulated case.

A similar kind of gain modulation has been previously used for multi-color operation and spectral control of the FEL output at LCLS [32]. In our scheme we add a modulation to the undulator field of the form $\Delta K(z)=\delta_{m} \sin k_{m} z$ with $\delta_{m}$ and $k_{m}$ the amplitude and wave number of the modulation (see Fig. 5, top). The amplitude is set to provide a phase shift that will generate destructive interference at the peak sideband frequency $\delta_{m} \sim \frac{1+K^{2}}{2 K^{2}} \frac{\lambda_{w}}{2 L_{s}}$. The modulation period $T_{m}=2 \pi / k_{m}$ is set to around one sideband "growth length" which we obtain from 1-D simulation as shown in Fig. 4, bottom. We show an example of the effect of gain modulation on the particle trapping in Fig. 5 and the sideband spectrum in Fig. 6. The parameters are the same as Table I with a $N_{u}=2 \times 10^{3}$ period undulator, a seed power 100 times larger than the shot noise power and a resonant phase of $\psi_{r}=\pi / 16$. The amplitude of the modulation is $\delta_{m}=2.2 \times 10^{-3}$ with a $2 \mathrm{~m}$ period. The example has a single modulation section of length 1.5 modulation periods starting at $N_{u}=750$ undulator periods. The trapping fraction (Fig. 5, bottom) shows a 15\% reduction around $N_{u}=800$ due to the resonant phase shifting during the modulation section. Following this region of particle loss, the trapping fraction remains constant in the rest of the undulator. This is in contrast with the unmodulated case which suffers from severe sideband-induced detrapping, losing around 50\% of the particles in the last 500 undulator periods. From the spectrum in Fig. 6 we see that the single modulation section reduces the relative sideband power from $55 \%$ to $4 \%$ of the total power compared to the unmodulated case. Furthermore, the peak spectral power also increases by $70 \%$ compared to the unmodulated case. The advantage of this method relative to methods previously mentioned is that it is readily applicable in any tapered FEL facility and requires no additional hardware. Numerical investigation of the effectiveness of this method with 3-D simulations will be pursued in future work.

\section{CONCLUSION}

We have presented an analysis of the physics of high gain high efficiency FELs in the 1-D limit discussing the key scalings and dependence of the output power on the tapering strategy (choice of resonant phase) and the initial conditions (unbunched vs pre-bunched beam). We have derived a simple scaling law to predict the peak output power highlighting the importance of electron trapping to the final output efficiency $P_{\text {out }} \propto f_{t}^{2}$. Comparing this formula to 1-D simulations we find good agreement for different cases starting from a cold beam with and without pre-bunching. The results display the advantage of pre-bunching in a high efficiency FEL due to the increased particle trapping in the post-saturation region. The main results from time independent simulations show that the efficiency can be increased by a factor of 2 to greater than 4 (depending on the prebunching seed power) compared to the unbunched case. Prebunching not only increases the peak efficiency but exhibits optimal energy extraction at larger resonant phase compared to the unbunched case. This faster extraction of energy is important for reducing the impact of harmful 3-D effects, specifically diffraction due to reduced optical guiding in tapered FELs with long undulators.

We have also presented an analysis of the 1-D sideband instability in high efficiency FELs and have discussed the scaling of the sideband growth rate as a function of the resonant phase. Through this discussion it is clear that it is advantageous to operate a tapered FEL at the largest possible resonant phase to suppress sideband growth by rapidly changing the synchrotron frequency along the undulator. Having a prebunched electron beam allows more aggressive (larger resonant phase) tapered FEL designs and therefore damps the generation of sidebands compared to the unbunched case. Finally, we have presented a method of sideband suppression by modulating the FEL gain via a controlled modulation of the undulator field. This is shown to significantly reduce the relative sideband/ total power ratio (over a factor of 10 in our example) and can be applied to high gain high efficiency FELs without the requirement of any additional hardware. 


\section{ACKNOWLEDGMENTS}

This work was partly supported by U.S. D.O.E. under Grants No. DE-SC0009983, No. DE-SC0009914 and by Small Business Innovative Research Contract No. DESC0017102.

\section{APPENDIX A: ALTERNATIVE DERIVATION OF THE EFFICIENCY SCALING}

Undulator tapering is required to match the electron energy loss from the interaction with the radiation (ponderomotive gradient) with the variation of the resonant energy profile along the undulator. For a constant period, helical undulator, the tapering equation for the normalized magnetic field amplitude can be written as [10]:

$$
\frac{d K}{d z}=-2 k_{w} K_{l} \sin \psi_{r}
$$

where $K_{l}=e E_{0} / m c^{2} k$ is the normalized vector potential of the radiation. Integration of Eq. (A1) is trivial in the case of low gain where we can assume the radiation is constant. When this is not the case we can take a second derivative and use Eq. (3) to get:

$$
\frac{d^{2} K}{d z^{2}}=-\left(\frac{2 k_{w}}{k}\right)^{3 / 2} \frac{e Z_{0} \bar{I}}{8 \pi \sigma_{e}^{2}} \sin \psi_{r}^{2} \frac{K}{\sqrt{1+K^{2}}} .
$$

This equation can be numerically integrated, or we can make the assumption that $K^{2} \gg 1$ which allows us to write an analytical expression for the tapering of the undulator:

$$
K=K_{0}-b z-\frac{c z^{2}}{2}
$$

where

$$
\begin{gathered}
b=2 k_{w} K_{l}(0) \sin \psi_{r} \\
c=\left(\frac{2 k_{w}}{k}\right)^{3 / 2} \frac{e Z_{0} \bar{I}}{8 \pi \sigma_{e}^{2}} \sin \psi_{r}^{2} .
\end{gathered}
$$

The efficiency can be written in terms of the known variation of $K$ assuming a constant fraction of trapped particles:

$$
\begin{aligned}
\eta & =f_{t}\left(1-\frac{\gamma_{r}}{\gamma_{0}}\right)=f_{t}\left(1-\sqrt{\frac{1+K^{2}}{1+K_{0}^{2}}}\right) \\
& \cong \frac{K_{0}}{1+K_{0}^{2}} f_{t}\left(b z+c z^{2} / 2\right)
\end{aligned}
$$

(a) Single Buncher

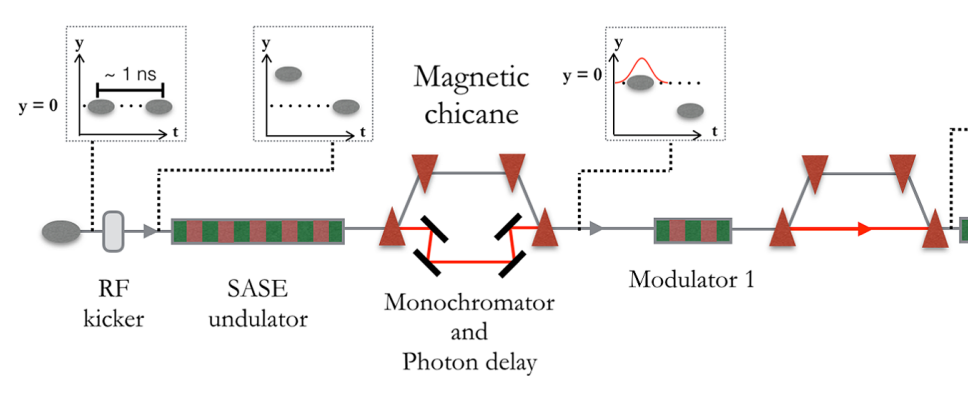

(b) Double Buncher

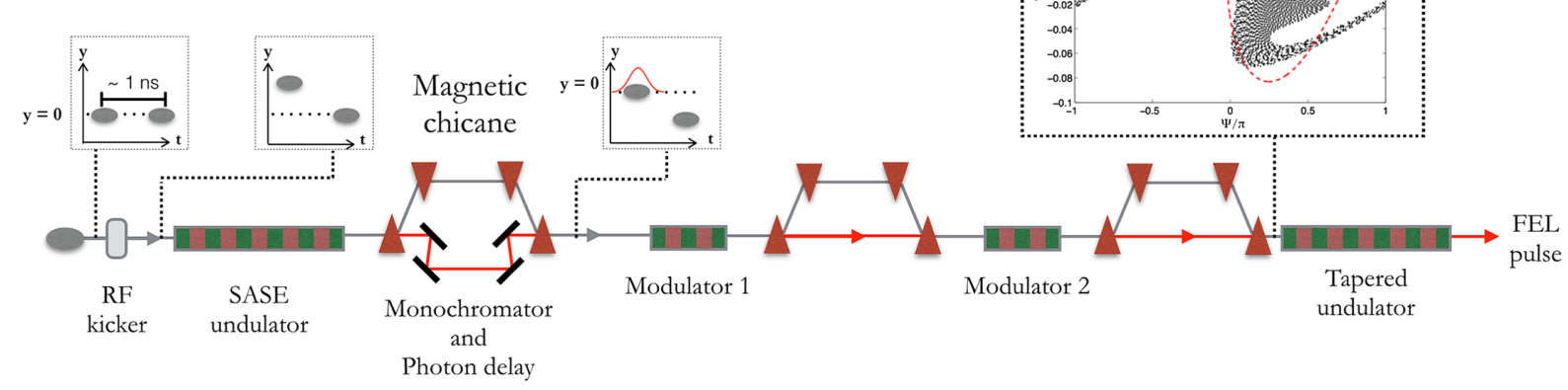

FIG. 7. (not to scale) Schematic of the double-buncher set-up used for a high gain high efficiency FEL. The modulator sections are typically short (exact length depending on the beam energy) compared to the SASE and tapered undulators. The trapping fraction at the start of the tapered section is large (66\% with the single buncher and $83 \%$ with the double-buncher) allowing quick and efficiency extraction of energy from the beam. 
where the last approximation holds only in the case of small efficiency (i.e., small relative variation for the normalized vector potential of the undulator). Equation (A6) is the same expression as Eq. (8) for the efficiency.

\section{APPENDIX B: PHASE-SPACE MODULATIONS FOR PREBUNCHING ELECTRON BEAMS}

We show a schematic of the single and double-buncher set-ups for a high gain high efficiency FEL in Fig. 7. The phase space transformations that take place between modulator 1 and the tapered undulator can be written following the notation of Ref. [33] and using the same treatment as Ref. [27] which discusses the double-buncher design for an inverse FEL accelerator. We note that predensity modulations to enhance the efficiency of other seeding schemes such as echo enabled harmonic generation have been discussed in Ref. [34]. Furthermore, a recent experiment demonstrating cascaded modulator-chicane pre-bunching in an inverse FEL has shown an increase in electron trapping from $\sim 60 \%$ (no pre-bunching) to $78 \%-$ $96 \%$ using a single pre-buncher [28]. The example in Fig. 7 shows how the double buncher captures a large fraction of the electron beam, $66 \%$ and $83 \%$ for $A=20$ and $\psi_{r}=\pi / 4$ in the single and double buncher cases, before the beam enters the tapered undulator. The modulator sections are typically a few undulator periods, short compared to the SASE and tapered undulator sections. For example, in the double-buncher case of Fig. 7(b) the first and second modulator are 5 and 11 undulator periods in length. The momentum compaction factors required to achieve the desired bunching are also practically feasible, with $R_{56}=$ $0.45 \mu \mathrm{m}$ and $R_{56}=0.15 \mu \mathrm{m}$ for the first and second chicanes. Note that the estimates assume helical undulators for the prebunching modulators and ignore laser diffraction.

[1] M. Roth and B. G. Logan, Advanced space power and propulsion based on lasers, Eur. Phys. J. Spec. Top. 224, 2657 (2015).

[2] K.-J. Kim, A. Zholents, M. Zolotorev, and N. Vinokurov, FEL options for power beaming, Nucl. Instrum. Methods Phys. Res., Sect. A 407, 380 (1998).

[3] Y. Socol, G. N. Kulipanov, A. N. Matveenko, O. A. Shevchenko, and N. A. Vinokurov, Compact 13.5-nm free-electron laser for extreme ultraviolet lithography, Phys. Rev. ST Accel. Beams 14, 040702 (2011).

[4] E. Hosler, O. Wood, and B. A. William, in Int'l Symposium Extreme UV Lithography (2014), http://euvlsymposium.lbl .gov/proceedings/2014.

[5] H. N. Chapman, P. Fromme, A. Barty, T. A. White, R. A. Kirian, A. Aquila, M. S. Hunter, J. Schulz, D. P. DePonte, $\mathrm{U}$. Weierstall et al., Femtosecond X-ray protein nanocrystallography, Nature (London) 470, 73 (2011).

[6] R. Neutze, R. Wouts, D. van der Spoel, E. Weckert, and J. Hajdu, Potential for biomolecular imaging with femtosecond X-ray pulses, Nature (London) 406, 752 (2000).
[7] M. Fuchs, M. Trigo, J. Chen, S. Ghimire, S. Shwartz, M. Kozina, M. Jiang, T. Henighan, C. Bray, G. Ndabashimiye et al., Anomalous nonlinear X-ray Compton scattering, Nat. Phys. 11, 964 (2015).

[8] R. Bonifacio, C. Pellegrini, and L. Narducci, Collective instabilities and high-gain regime in a free electron laser, Opt. Commun. 50, 373 (1984).

[9] N. M. Kroll, P. L. Morton, and M. Rosenbluth, Freeelectron lasers with variable parameter wigglers, IEEE J. Quantum Electron. 17, 1436 (1981).

[10] J. Duris, A. Murokh, and P. Musumeci, Tapering enhanced stimulated superradiant amplification, New J. Phys. 17, 063036 (2015).

[11] C. Emma, K. Fang, J. Wu, and C. Pellegrini, High efficiency, multiterawatt x-ray free electron lasers, Phys. Rev. Accel. Beams 19, 020705 (2016).

[12] T. J. Orzechowski, B. R. Anderson, J. C. Clark, W. M. Fawley, A. C. Paul, D. Prosnitz, E. T. Scharlemann, S. M. Yarema, D. B. Hopkins, A. M. Sessler et al., High-Efficiency Extraction of Microwave Radiation from a Tapered-Wiggler Free-Electron Laser, Phys. Rev. Lett. 57, 2172 (1986).

[13] Y. Jiao, J. Wu, Y. Cai, A. W. Chao, W. M. Fawley, J. Frisch, Z. Huang, H.-D. Nuhn, C. Pellegrini, and S. Reiche, Modeling and multidimensional optimization of a tapered free electron laser, Phys. Rev. ST Accel. Beams 15, 050704 (2012).

[14] A. Mak, F. Curbis, and S. Werin, Model-based optimization of tapered free-electron lasers, Phys. Rev. ST Accel. Beams 18, 040702 (2015).

[15] E. A. Schneidmiller and M. V. Yurkov, Optimization of a high efficiency free electron laser amplifierPhys. Rev. ST Accel. Beams 18, 030705 (2015).

[16] N. Sudar, P. Musumeci, J. Duris, I. Gadjev, M. Polyanskiy, I. Pogorelsky, M. Fedurin, C. Swinson, K. Kusche, M. Babzien et al., High Efficiency Energy Extraction from a Relativistic Electron Beam in a Strongly Tapered Undulator, Phys. Rev. Lett. 117, 174801 (2016).

[17] Perave: a Matlab-based 1-D FEL code http://pbpl.physics .ucla.edu/Computing/Code_Development/Perave.

[18] N. Kroll and M. Rosenbluth, in Free Electron Generators of Coherent Radiation, edited by S. Jacobs, H. Pilloff, M. Sargent, M. Scully, and R. Spitzer (Springer, Berlin Heidelberg, 1979), p. 147-174.

[19] D. C. Quimby, J. M. Slater, and M. Wilcoxon, Sideband suppression in free-electron lasers with multiple synchrotron periods, IEEE J. Quantum Electron. 21, 979 (1985).

[20] S. Riyopoulos and C. M. Tang, Chaotic electron motion caused by sidebands in free electron lasers, Phys. Fluids 31, 3387 (1988).

[21] W. M. Fawley, "Optical guiding" limits on extraction efficiencies of single-pass, tapered wiggler amplifiers Nucl. Instrum. Methods Phys. Res., Sect. A 375, 550 (1996),

[22] C. Emma, J. Wu, K. Fang, S. Chen, S. Serkez, and C. Pellegrini, Terawatt x-ray free-electron-laser optimization by transverse electron distribution shaping, Phys. Rev. ST Accel. Beams 17, 110701 (2014).

[23] R. Bonifacio, C. F., G. Cerchioni, L. De Salvo Sousa, P. Pierini, and N. Piovella, Physics of the high-gain FEL and 
superradiance, Riv. Nuovo Cimento Soc. Ital. Fis. 13, 1 (1990).

[24] K. J. Kim, Z. Huang, and R. Lindberg, Synchrotron Radiation and Free-Electron Lasers: Principles of Coherent X-Ray Generation (Cambridge University Press, Cambridge, England, 2017).

[25] R. Brau and C. Cooper, in Free Electron Generators of Coherent Radiation, edited by S. Jacobs, H. Pilloff, M. Sargent, M. Scully, and R. Spitzer (Springer, Berlin Heidelberg, 1979), p. 647.

[26] S. Y. Lee, Accelerator Physics (World Scientific Press, Singapore, 2012).

[27] N. Sudar, P. Musumeci, and J. Duris (to be published); available online, http://www.sciencedirect.com/science/ article/pii/S0168900217301924.

[28] N. Sudar, P. Musumeci, I. Gadjev, Y. Sakai, S. Fabbri, M. Polyanskiy, I. Pogorelsky, M. Fedurin, C. Swinson, K. Kusche et al. (to be published), https://arxiv.org/abs/1708 .05456 .

[29] I. Ben-Zvi, K. Yang, and L. Yu, The "fresh-bunch" technique in FELS, Nucl. Instrum. Methods Phys. Res., Sect. A 318, 726 (1992).
[30] C. Emma, A. Lutman, M. Guetg, J. Kryziwinski, A. Marinelli, J. Wu, and C. Pellegrini, Experimental demonstration of fresh bunch self-seeding in an X-ray free electron laser, Appl. Phys. Lett. 110, 154101 (2017).

[31] C. Emma, Y. Feng, D. C. Nguyen, A. Ratti, and C. Pellegrini, Compact double-bunch $\mathrm{X}$-ray free electron lasers for fresh bunch self-seeding and harmonic lasing, Phys. Rev. Accel. Beams 20, 030701 (2017).

[32] A. Marinelli, A. A. Lutman, J. Wu, Y. Ding, J. Krzywinski, H.-D. Nuhn, Y. Feng, R. N. Coffee, and C. Pellegrini, Multicolor Operation and Spectral Control in a GainModulated X-Ray Free-Electron Laser, Phys. Rev. Lett. 111, 134801 (2013).

[33] G. Stupakov, Using the Beam-Echo Effect for Generation of Short-Wavelength Radiation, Phys. Rev. Lett. 102, 074801 (2009).

[34] C. Feng, D. Wang, and Z. Zhao, in Proceedings of the 32nd Free Electron Laser Conference, Malmö, Sweden (Max-lab, Sweden, 2010). 